Research Paper

\title{
Diet- and Genetically-induced Obesity Produces Alterations in the Microbiome, Inflammation and Wnt Pathway in the Intestine of $\mathrm{Apc}^{+/ 1638 \mathrm{~N}}$ Mice: Comparisons and Contrasts
}

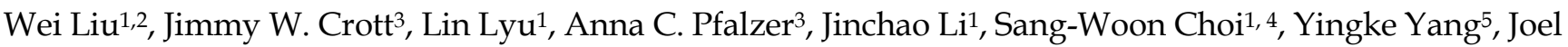 \\ B. Mason ${ }^{3,6}$, Zhenhua Liu ${ }^{1,3} \bowtie$ \\ 1. Nutrition and Cancer Prevention Laboratory, School of Public Health and Health Sciences, University of Massachusetts, Amherst, USA. \\ 2. Institute of Food Science and Technology, Shandong Academy of Agricultural Sciences, Jinan, China. \\ 3. Jean Mayer USDA Human Nutrition Research Center on Aging, Tufts University, Boston, USA. \\ 4. CHA Cancer Research Center, CHA University, Seoul, Korea. \\ 5. Department of Molecular Medicine, Hunan University, Changsha, China. \\ 6. Tufts Medical Center Cancer Center, Tufts University, Boston, USA. \\ $\square$ Corresponding author: Zhenhua Liu, Ph.D. Nutrition and Cancer Prevention Laboratory, School of Public Health and Health Sciences, University of \\ Massachusetts, Amherst, 100 Holdsworth Way, Amherst, MA, 01003 Email: zliu@nutrition.umass.edu; Tel: 1-413-545-1075; Fax: 1-413-545-1074.
}

() Ivyspring International Publisher. Reproduction is permitted for personal, noncommercial use, provided that the article is in whole, unmodified, and properly cited. See http://ivyspring.com/terms for terms and conditions.

Received: 2016.04.10; Accepted: 2016.06.29; Published: 2016.08.12

\begin{abstract}
Obesity is an established risk factor for colorectal cancer (CRC). Our previous study indicated that obesity increases activity of the pro-tumorigenic Wnt-signaling. Presently, we sought to further advance our understanding of the mechanisms by which obesity promotes CRC by examining associations between microbiome, inflammation and Wnt-signaling in $\mathrm{Apc}^{+/ 1638 \mathrm{~N}}$ mice whose obesity was induced by one of two modalities, diet- or genetically-induced obesity. Three groups were employed: $A \mathrm{Ac}^{+/ 1638 \mathrm{~N}} \mathrm{Lepr}^{+/+}$fed a low fat diet $(10 \% \mathrm{fat}), \mathrm{Apc}^{+/ 1638 \mathrm{~N}} \mathrm{Lepr}^{+/+}$fed a high fat diet $\left(60 \%\right.$ fat, diet-induced obesity), and $\mathrm{Apc}^{+/ 1638 \mathrm{~N}} \mathrm{Lepr}^{\mathrm{db} / \mathrm{db}}$ fed a low fat diet (genetically-induced obesity). All animals received diets for 16 weeks from 8 to 24 weeks of age. The abundance of 19 bowel cancer-associated bacterial taxa were examined by real-time PCR. The abundance of Turicibacter and Desulfovibrio decreased, but $F$. prausnitizii increased, in diet-induced obese mice $(\mathrm{p}<$ $0.05)$. In contrast, in genetically-induced obese mice, Bifidobacterium, A. muciniphila and E. rectale decreased, but Peptostrptococcus, and E. coli increased ( $p<0.05)$. Both diet- and genetically-induced obesity altered the expression of genes involved in bacterial recognition (MyD88) and increased inflammation as indicated by elevated levels of cytokines (IFN $\gamma$ and TNF- $\alpha$ for genetically-induced obesity, and IL-6 for diet-induced obesity). The elevated inflammation was associated with altered expression of genes that are integral components of the Wnt-signaling cascade in a fashion indicating its activation. These findings demonstrate that the composition of the small intestinal microbiome is affected differently in diet- and genetically-induced obesity, but both are associated with elevated intestinal inflammation and alterations of the Wnt pathway towards enhancing tumorigenesis.
\end{abstract}

Key words: Obesity, Microbiome, Inflammation, Wnt pathway, Intestinal cancer.

\section{Introduction}

In recent decades, there has been a striking increase of obesity in both developed countries and urban regions of developing countries. Obesity is now a major global public health issue and a further increase of $\sim 50 \%$ by 2030 is predicted $(1,2)$. Epidemiological studies have suggested obesity is an 
important risk factor for colorectal cancer (CRC), with $50-100 \%$ increased risks for women and men who have a BMI $\geqslant 30$ (3). Animal studies are in agreement with both diet- and genetically-induced obesity promoting intestinal tumorigenesis $(4,5)$. CRC is a major cause of death: $\sim 50,000$ individuals annually in the US and $\sim 700,000$ worldwide $(6,7)$, thus understanding all of the risk factors for CRC and identifying means to combat them is of the utmost importance.

It is well-known that obesity induces chronic low-grade inflammation in the adipose tissue as well as systemically $(8,9)$, and recent animal studies-including our own-indicate that an inflammatory milieu resides in the colon as well (10, 11). Our prior study demonstrates that obesity-driven inflammation, exemplified by an increased production of inflammatory cytokine TNF- $\alpha$, is associated with alterations of several key components (GSK3 $\beta$ and $\beta$-catenin) of the Wnt-signaling pathway, (10) the over-activation of which is thought to be an early integral step in $90 \%$ of human CRCs $(12,13)$.

While increasing evidence indicates that obesity-driven chronic intestinal inflammation plays an important role in colorectal tumorigenesis, the origin of this inflammation remains elusive. Recent studies suggest that alterations in the composition and diversity of intestinal microbiome is an important characteristic of obesity (14). Animal studies indicate that both diet- (15) and genetically-induced (16) obesity alters the composition of microbiome characterized by an expansion of Firmicutes and reduction in Bacteroidetes at the phylum level. A disruption of the 'normal' microbial community is thought to increase the risk of pathogen infection and to promote inflammation (17).

To understand the role of gut microbiome in linking obesity with inflammation, Wnt pathway and tumorigenesis, we sought to examine how high fat diet and obesity per se drive gut dysbiosis, promote intestinal inflammation, activate the Wnt pathway, and thereby enhance intestinal tumorigenesis using the $\mathrm{Apc}^{+/ 1638 \mathrm{~N}}$ mouse model, the tumorigenic phenotype of which is particularly sensitive to dietary modifications $(18,19)$.

\section{Experimental Methods}

\section{Animal study}

The protocol for this study was approved by the Institutional Animal Care and Use Committee of the Jean Mayer USDA Human Nutrition Research Center on Aging at Tufts University. A detailed description of the protocol has previously been reported (20). Briefly, in order to study how obesity influences the intestinal microbiome and tumorigenesis, we utilized both high fat diet-induced $(60 \% \mathrm{kcal}$ fat $)$ and genetically-induced (Lepr ${ }^{\mathrm{db} / \mathrm{db}}$ ) obese models (21). To study intestinal tumorigenesis, we utilized the $\mathrm{Apc}^{+/ 1638 \mathrm{~N}}$ model (22). Though this model develops tumors predominantly in the small intestine rather than the colorectum, it is the nature of other genetically-engineered rodent models of CRC such as the widely used $\mathrm{Apc}^{\mathrm{Min}}$ mouse. Nevertheless, the $\mathrm{Apc}^{+/ 1638 \mathrm{~N}}$ model has a mildly tumorigenic phenotype that sensitively responds to dietary modification and the predilection to developing these small intestinal tumors responds to dietary perturbations in the same manner that diet modifies CRC risk in the human, underscoring the relevance of this model to human colonic tumorigenesis $(18,19)$. Since the tumors predominantly occur in the small intestine in this mouse model, the small intestinal microbial constitution is more reflective of the tumor microenvironment, and therefore in this paper we focused on the microbial composition, inflammatory status and expression of Wnt pathway-specific genes in the small intestine as opposed to the large intestine.

Three groups with 2 different genotypes and 2 dietary treatments were used in this study:

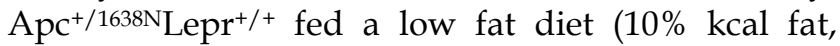
Apc-LF), Apc ${ }^{+/ 1638 N} \mathrm{Lepr}^{+/+}$fed a high fat diet $(60 \%$ kcal fat, Apc-HF), and Apc ${ }^{+/ 1638 N}$ Lepr $^{\mathrm{db} / \mathrm{db}}$ fed a low fat diet (Apc-DB). Animals (9-12 mice/group) received diets for 16 weeks from 8 to 24 weeks of age. The compositions of experimental diets were previously described. (20) Mice were housed in a controlled environment (inverted 12-h daylight cycle) with ad libitum access to food and water.

After 16 weeks on diet, mice were sacrificed, the abdomen was opened, and small intestines were removed onto ice-cold glass plates. Samples of the intestinal contents were harvested, frozen in liquid nitrogen, and stored at $-80^{\circ} \mathrm{C}$ for further microbial abundance analysis. After rinsing thoroughly with ice-cold PBS with protease inhibitors (Roche, Indianapolis, IN), the small intestinal tumors were characterized under a dissecting microscope. The remaining normal-appearing small intestinal mucosa was harvested by gentle scraping with microscope slides at $0^{\circ} \mathrm{C}$, frozen in liquid nitrogen, and stored at $-80{ }^{\circ} \mathrm{C}$ for later analysis of inflammatory cytokines and expression of Wnt pathway-specific genes.

\section{Quantification of small intestinal microbiome}

The relative abundance of 19 bacterial taxa, which have been reported to be associated with the development of colonic adenomas and cancers in mice and/or humans (23), were determined by real-time PCR. Most of primers used in this study have been 
previously validated; the remaining primers were designed on the basis of $16 \mathrm{~S}$ rDNA gene sequences available from the GenBank database and Ribosomal Database Project database (http://rdp.cme.msu. edu/). Primers were synthesized commercially (Invitrogen Life Technologies, Carlsbad, CA), and sequences are listed in Supplementary Data (Table S1).

Bacterial DNA was extracted from small intestinal contents using the QIAamp DNA Stool Mini Kit (Qiagen, Valencia, CA) according to the manufacturer's instructions. The concentration and purity of extracted DNA was determined using the NanoDrop-2000 spectrophotometer (Thermo Scientific, Waltham, MA). Real-time PCR assays were on the ViiA ${ }^{\mathrm{TM}} 7$ Real-Time PCR System (Applied Biosystems, Foster City, CA). PCR conditions were 15 min at $95^{\circ} \mathrm{C}$, followed by 40 cycles of $95^{\circ} \mathrm{C}$ for $1 \mathrm{~min}$, $30 \mathrm{~s}$ at the annealing temperature, and then $72^{\circ} \mathrm{C}$ for 1 min.

\section{Inflammatory cytokine assay}

The small intestine mucosa scrapings (50 100 $\mathrm{mg})$ were mixed with $400 \mu \mathrm{L}$ lysis buffer $(150 \mathrm{mM}$ $\mathrm{NaCl}, 20 \mathrm{mM}$ Tris, $1 \mathrm{mM}$ EDTA, 1\% Triton X-100, $\mathrm{pH}$ 7.5) and phosphatase and protease inhibitors (Sigma, St. Louis, MO) were added to inhibit degradation. Samples were homogenized and then centrifuged at $4^{\circ} \mathrm{C}$ at $12000 \mathrm{rpm}$ for $30 \mathrm{~min}$, the protein fraction was collected, and the total protein concentrations were measured in the extract using the Bradford assay. Concentrations of IL-1 $\beta$, TNF- $\alpha$, IL- 6 and IFN $\gamma$ were determined using the Proinflammatory Panel 1 (mouse) V-PLEXTM Kit and QuickPlex SQ 120 (Mesoscale Discovery, Rockville, MD) according to manufacturer's protocols. Cytokines are expressed as $\mathrm{ng} / \mathrm{mg}$ protein.

\section{Gene expression profile}

The relative expressions were determined for 6 genes involved in bacterial recognition and inflammatory processes, and 16 genes selected from the Wnt pathway cascade including genes of Wnt ligands and Wnt antagonists, receptors and signaling transduction genes, and the downstream target genes. Briefly, total RNA was extracted from the small intestine with Trizol (Invitrogen, Carlsbad, CA), and cDNA was synthesized with SuperScript III (Invitrogen, Carlsbad, CA, USA). Real-time PCR was performed on the ViiA ${ }^{\mathrm{TM}} 7$ Real-Time PCR System (Applied Biosystems, Foster City, CA). Primer sequences were listed in supplementary Data (Table S2).

\section{Data analysis}

Data are expressed as means \pm SEM. Data analysis was performed using SAS (Version 9.4, SAS Institute, Cary, NC) or GraphPad Prism ${ }^{\mathrm{TM}}$ (Version 6, GraphPad Software, La Jolla, CA). Comparisons between groups were made with a T-test or ANOVA and associations were assessed by Pearson's Correlation. Significance was accepted when $p<0.05$, and a false discovery rate cutoff of $\mathrm{q}<0.2$ was used when multiple comparisons were conducted. Heatmaps were created based on transformed z-score values using HemI software (24).

For the bacterial data analysis, the abundance of specific bacterial taxa was normalized to total bacteria $\left(\Delta \mathrm{Ct}=\mathrm{Ct}_{\text {specific }}-\mathrm{Ct}_{\text {total }}\right)$, and for the gene expression data analysis, the expression of each gene was normalized to the housekeeping gene GAPDH ( $\mathrm{Ct}_{\text {target }}$ gene- $\left.\mathrm{Ct}_{\mathrm{GAPDH}}\right)$. Statistical analyses were performed based on $\Delta \mathrm{Ct}$. The relative abundance of specific bacterial taxa or relative gene expression in diet- and genetically-induced obese groups (Apc-HF and Apc-DB, respectively) comparing to the control group (Apc-LF) were reported as $2^{-\Delta \Delta \mathrm{Ct}}$, where $\Delta \Delta \mathrm{Ct}=\Delta \mathrm{Ct}_{\mathrm{Apc}-\mathrm{HF}-}-\Delta \mathrm{Ct}_{\mathrm{Apc}-\mathrm{LF}}$ or $\Delta \Delta \mathrm{Ct}=\Delta \mathrm{Ct}_{\mathrm{Apc}-\mathrm{DB}}-\Delta \mathrm{Ct}_{\mathrm{Apc}-\mathrm{LF}}$.

\section{Results}

\section{Physiological characteristics and intestinal tumors}

Physiological and tumor data have been previously reported (20). Briefly, the lack of leptin receptor in the genetically-induced obese Lepr $\mathrm{db} / \mathrm{db}$ mouse model (Apc-DB) resulted in significantly increased body weight in both males $(64.8 \%)$ and females $(113 \%)$ when compared to the wildtype control animals (Apc-LF) even though both groups were fed a low fat diet $(10 \% \mathrm{kcal}$ fat $)(p<0.01)$. The high fat diet $(60 \%$ kcal fat, Apc-HF) also induced significant increases $(p<0.05)$ in body weight when compared to the low fat diet group (Apc-LF), although the magnitude in both males $(44.3 \%)$ and females $(29.2 \%)$ were lower than genetically-induced obesity. Fat mass was also significantly higher in Apc-DB mice compared to Apc-HF and Apc-LF mice, and Apc-HF mice had significantly greater body fat than Apc-LF mice (20). Tumor incidence, the proportion of animals in each group possessing tumors, was $33 \%, 67 \%$ and $100 \%$ in Apc-LF, Apc-HF and Apc-DB mice, respectively $(p<0.01)$. A similar significant stepwise increase in tumor multiplicity (number of tumors harbored by each animal) and tumor burden (total volume of tumors in each animal) was also observed (20). 


\section{Small intestinal microbiome in diet- or genetically-induced obese mice}

Among the 19 cancer-related bacterial genera or species studied, the abundance of 4 taxa was altered by diet-induced obesity, while 5 different taxa were altered by genetically-induced obesity $(\mathrm{p}<0.05)$ (Fig. 1). Specifically, the genera Desulfibrio and Turicibacter and species C.leptum were reduced while the species F.prausnitzii was elevated by high fat intake. The genus Bifidobacterium, and the species A. municiphila and E. rectale were decreased while genus Peptostreptococcus and species E.coli were increased by genetically-induced obesity. Very interestingly, none of these taxa were significantly altered by both modalities of obesity (Fig. 2). When a Pearson's correlation analysis was performed between the abundance of identified bacterial taxa and the body weight, statistically significant correlations $(p<0.05)$ were observed mainly for those bacterial taxa whose abundance was altered by genetically-induced obesity, but not for diet-induced obesity (Table 1).

Table 1. Correlations between the abundance of bacteria and body weight.

\begin{tabular}{llll}
\hline Bacteria & $\begin{array}{l}\text { Relative } \\
\text { Coefficience (R) }\end{array}$ & Bacteria & $\begin{array}{l}\text { Relative } \\
\text { Coefficience (R) }\end{array}$ \\
\hline At the genus level & \multicolumn{3}{c}{ At the species level } \\
\hline Bifidobacterium & $-0.408^{*}$ & A.muciniphila & $-0.390 *$ \\
Desulfovibrio & -0.165 & C.leptum & -0.021 \\
Pepstreptococcus & $0.428^{*}$ & E.rectale & $-0.435 *$ \\
Turicibacter & 0.104 & E.coli & $0.439 *$ \\
& & F.prausnitizii & -0.225
\end{tabular}

* Indicates a significant correlation $(p<0.05)$. Negative coefficient values indicate a reverse correlation, whereas positive coefficient values indicate a positive

correlation. The correlation analyses were performed on all experimental animals.

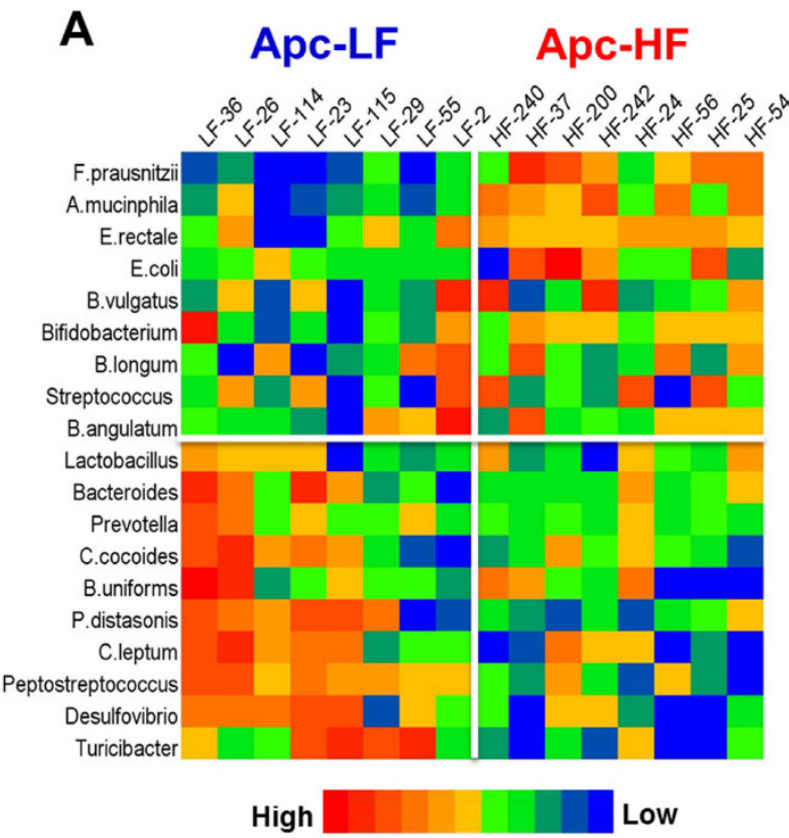

Diet-induced Obesity:

Genus Level: Desulfovibrio, Turicibacter Species Level: C.leptum, F.prausnitizii
B

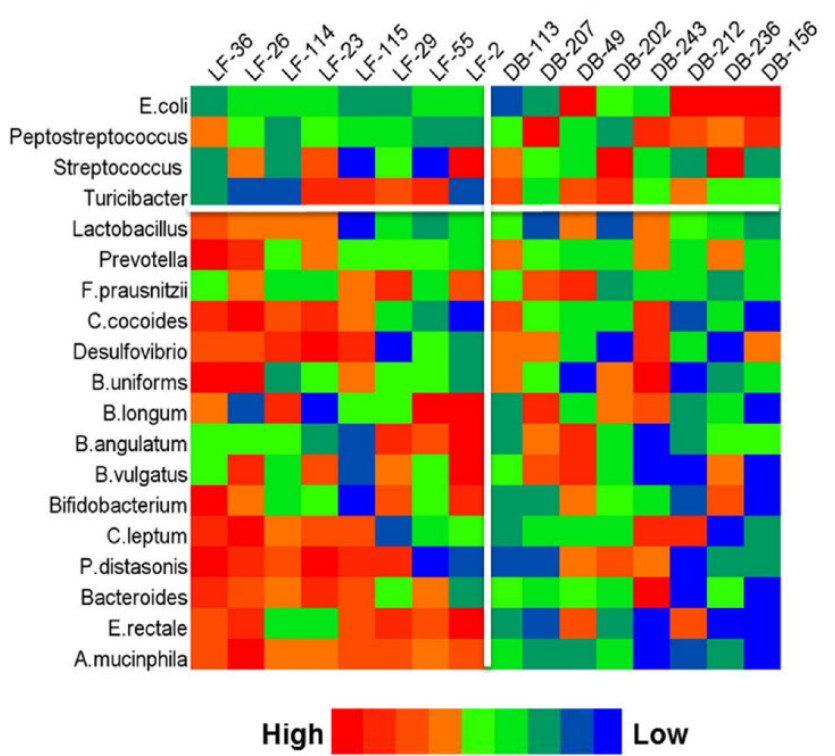

Genetically-induced Obesity:

Genus Level: Bifidobacterium, Pepstreptococcus Species Level: A.muciniphila, E.coli, E.rectale

Figure 1. Heatmaps of microbiome in the small intestine of mice. The microbial taxa are ranked based on the relative abundance from the high to the low scale. Microbial taxa with increased abundance appear in the upper boxes (above the white line) and taxa with decreased abundance are in the lower boxes (below the white line). Those taxa whose relative abundance were significantly different in the obese state compared to the lean are listed at the bottom. A) Heatmap for the comparison between the Apc ${ }^{+/ 1638 N L e p r^{+/+}}$fed a high fat diet $\left(60 \% \mathrm{kcal}\right.$ fat, Apc-HF) and the Apc ${ }^{+/ 1638 N L e p r^{+/+}}$fed a low fat diet (10\% kcal fat, Apc-LF). B) Heatmap for the comparison between the Apc ${ }^{+/ 1638 N L e p r d b / d b}$ fed a low fat diet $\left(10 \%\right.$ kcal fat, Apc-DB) and the Apc ${ }^{+/ 1638 N L e p r} r^{+/+}$fed a low fat diet $(10 \%$ kcal fat, Apc-LF). 

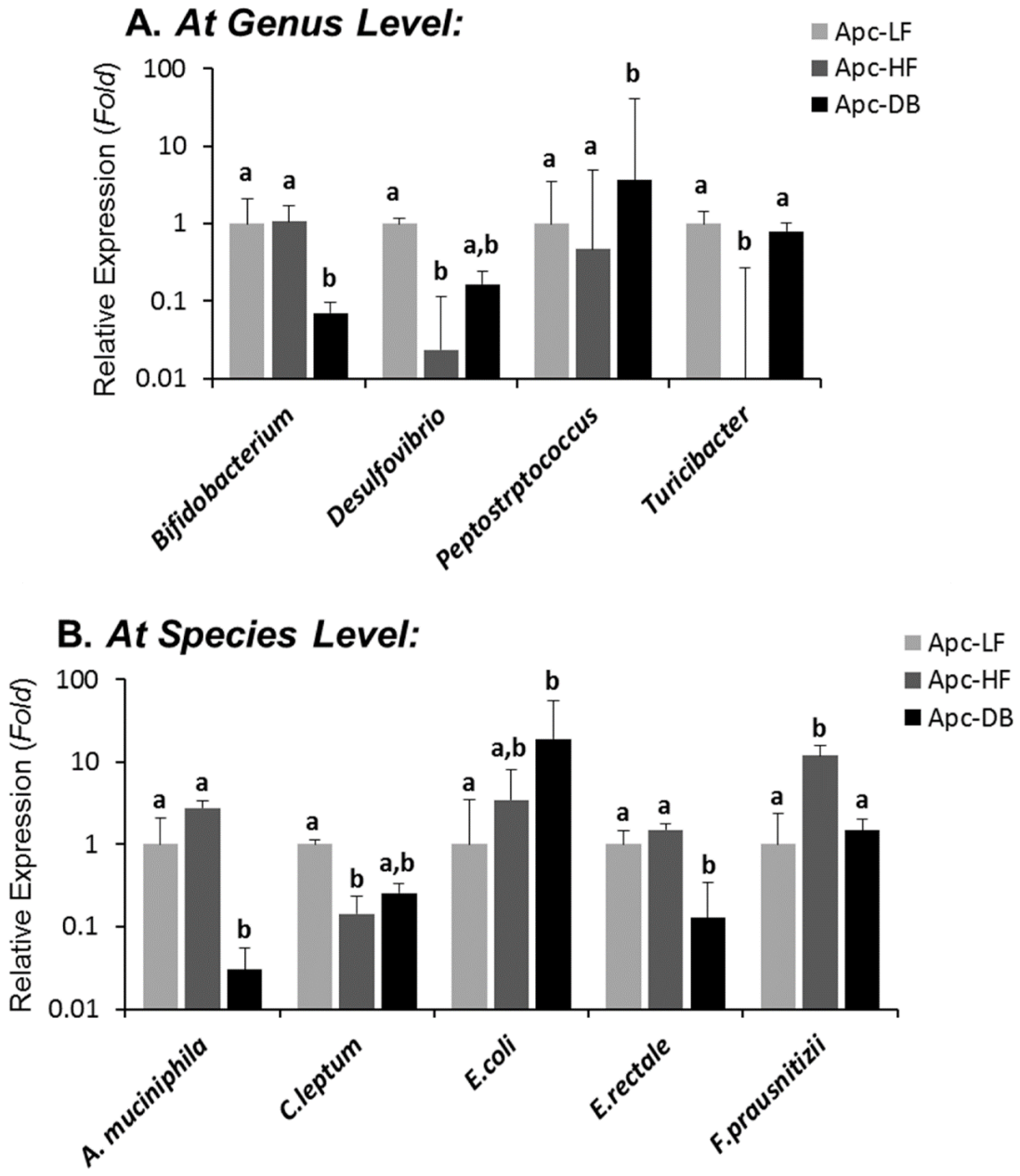

Figure 2. Differential influences of high fat- (60\% kcal fat, Apc-HF) and genetically-induced (Apc-DB) obesity on the intestinal cancer-related microbiome compared to the low fat control group (10\% kcal fat, Apc-LF). Altered abundances of Turicibacter and F. prausnitzii are present in high fat diet-induced obesity, and altered abundances for Bifidobacterium, Peptostreptococcus, A. mucinphila and E. rectale are present in genetically-induced obesity. A) At the genus level. B) At the species level.

\section{Transcriptional expression of genes involved in bacterial recognition and pro-inflammatory processes}

Among the 6 genes involved in bacterial recognition and pro-inflammatory processes, the transcriptional expression of myeloid differentiation factor 88 (MyD88), a cytoplasmic molecule which plays a central role in immune response to many bacterial cell components including lipopolysaccharide, peptidoglycan, and lipopeptide $(25,26)$, was significantly elevated in both diet-and genetically-induced obesity groups when comparing to the low fat diet control group $(\mathrm{p}<0.05)$. In addition, the expression of NFKB1, the encoded protein of which plays a central role in determining the specificity of $N F \kappa B$ signaling, was significantly higher in the Apc-HF group whereas the expression of Nod2, which encodes a protein that recognizes bacterial peptidoglycans and links recognition to the appropriate immune response, was low in the Apc-DB group compared to the Apc-HF group (Fig. $3 A$ ). When a Pearson's correlation analysis was performed for those genes involved in bacterial recognition and pro-inflammatory processes including inflammatory cytokines and microbial taxa, we detected a number of correlations among them, and the correlations for the two genera, Bifidobacterium and Turicibacter, were shown in Table 2.

Table 2. The correlation of transcriptional expression between genes involved in bacterial recognition and pro-inflammatory process with the abundance of microbial taxa.

\begin{tabular}{llll}
\hline & & Bifidobacterium & Turicibacter \\
\hline $\begin{array}{l}\text { Genes involved in } \\
\text { bacterial }\end{array}$ & MyD-88 & $\mathrm{r}=0.61(\mathrm{p}=0.044)$ & $\mathrm{NS}$ \\
$\begin{array}{l}\text { Recognition } \\
\begin{array}{l}\text { Genes involved in } \\
\text { pro- }\end{array}\end{array}$ & NF- $\mathrm{\kappa B} 1$ & $\mathrm{NS}$ & $\mathrm{r}=-0.56(\mathrm{p}=0.020)$ \\
$\begin{array}{l}\text { inflammation } \\
\text { TNF- } \mathrm{a}\end{array}$ & $\mathrm{r}=-0.69(\mathrm{p}=0.013)$ & $\mathrm{rS}=-0.61(\mathrm{p}=0.007)$ \\
\hline
\end{tabular}




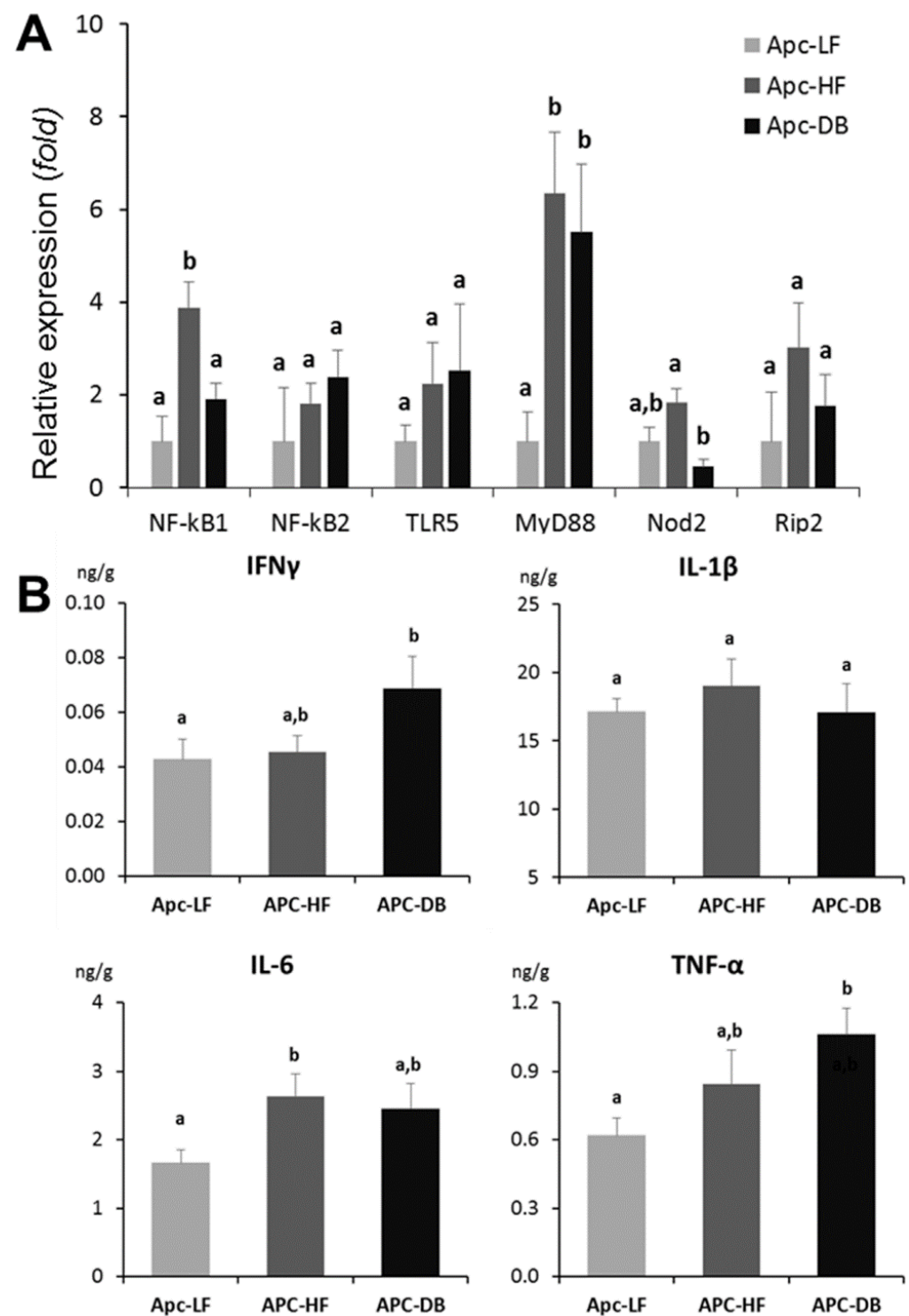

Figure 3. Impact of obesity on inflammation in the small intestine of mice. A) Impact of obesity on the expression of genes involved in bacterial recognition and pro-inflammatory processes in the small intestine of mice. B) Impact of obesity on inflammatory cytokines in the small intestine of mice. Apc-LF:

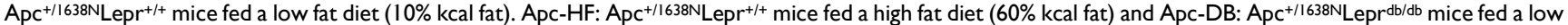
fat diet (10\% kcal fat).

\section{Intestinal inflammatory cytokine profile}

Among the 4 inflammatory cytokines examined (IFNY IL-1 $\beta$, IL-6 and TNF-a), IFNY and TNF-a were significantly increased $(72 \%$ and $60 \%$ respectively, $p<$ $0.05)$ in the genetically-induced obesity group (Apc-DB) when comparing to the Apc-LF group, whereas IL-6 was significantly increased $(58 \%$, p < 0.05 ) in the diet-induced obesity group (Apc-HF). Based on the inflammatory cytokine profile (Fig. 3B), both diet- and genetically-induced obesity resulted in a low-grade inflammation in the small intestine as indicated by numerical or statistically significant elevattions of inflammatory cytokine protein levels. The magnitudes of cytokine elevation for the genetically-induced obesity group were relatively higher than for the diet-induced group.

\section{Wnt pathway-specific gene expression profile}

We assessed the expression of 16 genes selected from Wnt pathway cascade including genes of Wnt ligands and Wnt antagonists, receptors and signaling transduction genes and the downstream target genes (Fig. 4A). Among the 16 genes examined, we observed 7 genes whose expressions were significantly elevated $(\mathrm{p}<0.05)$ by either diet- or genetically-induced obesity or both (Supplementary Materials, Table S2). Since the expression patterns between the diet- and genetically-induced groups are not significantly different when compared to the low fat diet control group, we combined the Apc-HF and Apc-DB groups 
to generate the heatmap. Among the 7 genes identified, three are the Wnt pathway downstream target genes, three are receptors and signaling transduction genes, and only 1 gene $(W n t 5 A)$ is among the Wnt ligands and Wnt antagonists group (Fig. 4B).

Pearson's correlations of transcriptional expression were then performed between MyD88, $N F \kappa B 1$ and Nod2, the 3 genes involved in bacterial recognition and pro-inflammatory processes and, with genes within the Wnt pathway cascade. We observed that multiple Wnt pathway genes correlated with MyD88 and NFkB1 (Table 3), and this was particularly true for downstream target genes and signaling transduction genes. Correlations between MyD88 and NFkB1 with C-Myc and Cyclin D1, the 2 critical Wnt pathway downstream oncogenes are shown in Fig. 4C.

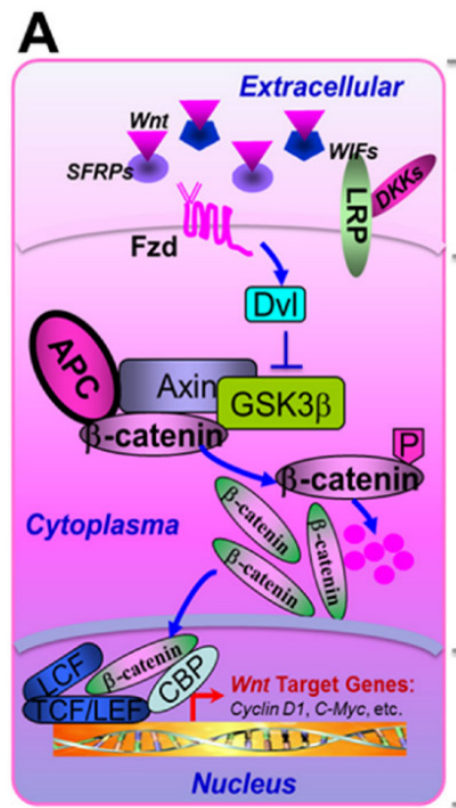

B

Wnts \& Wnt Antagonists: Wnt5a, SFRP1, SFRP5, etc.

Receptors \& Signalling Transduction Genes: Dvl, Apc, GSK36, 8-Catenin, Axin, Akt, etc.

Wnt Target Genes: C-Myc, Cyclin D1, JNK1, C-Jun, P53, Axin2, etc.
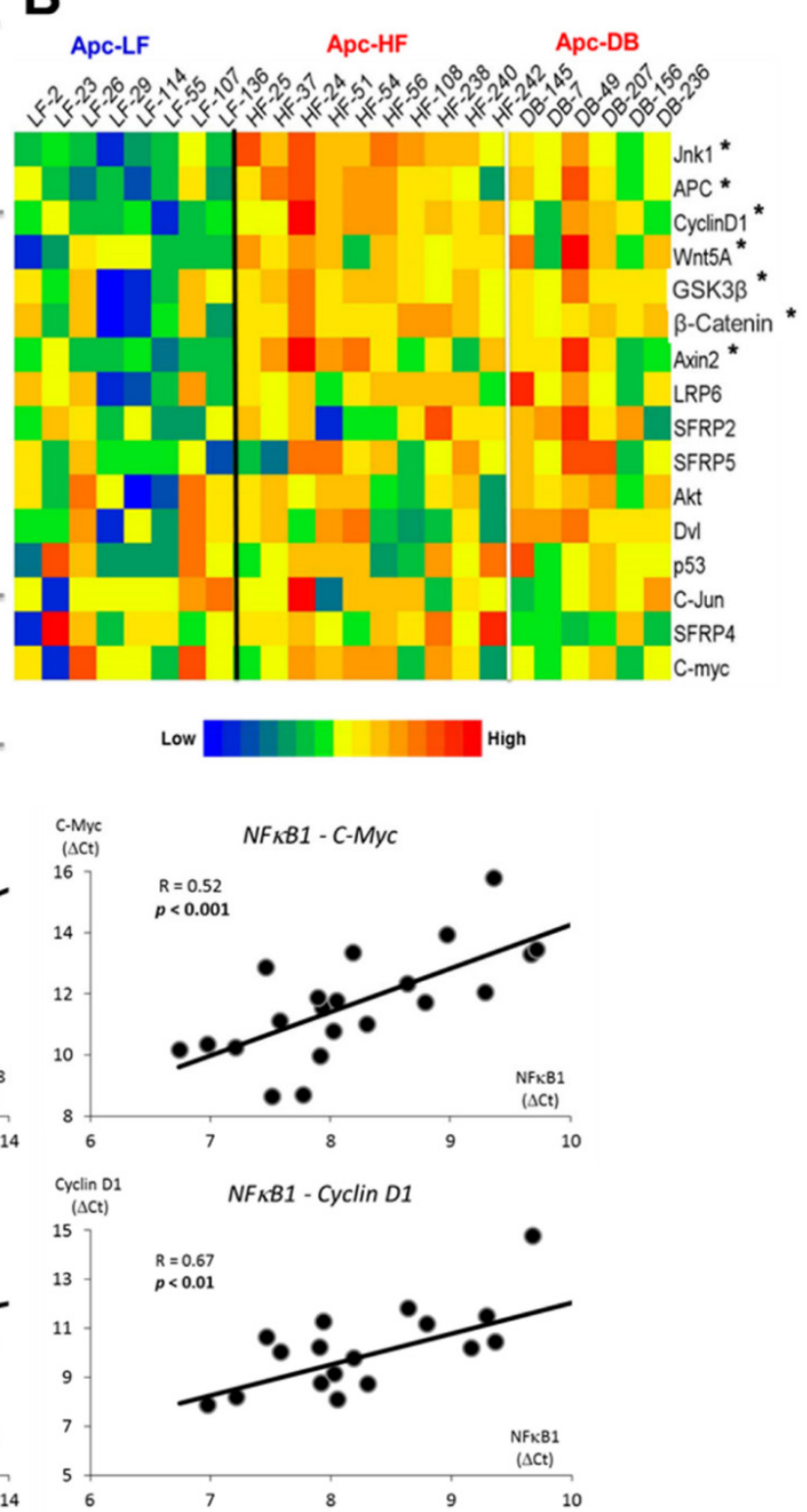

Figure 4. Effects of obesity on Wnt pathway-specific gene expression. A) Wnt-signaling pathway. 16 genes were selected, including Wn ligands and Wnt antagonists, receptors, signaling transduction genes and downstream target genes. B) Heatmap for the relative expression of Wnt pathway-specific genes examined in the small intestine of mice. The genes are ranked based on the relative expression, from the high to the low. C) Correlations between gene expression of MyD88 and $N F K B$ I, genes involved in bacterial recognition and inflammation, with C-Myc and Cyclin DI, downstream targets of the Wnt pathway. The expression patterns between the diet- and genetically-induced groups are not significantly different when comparing to the low fat diet control group. Therefore, the Apc-HF and Apc-DB groups were combined to generate the heatmap. Apc-LF: Apc $+/ 1638 \mathrm{Lepr}^{+/+}$mice fed a low fat diet (10\% kcal fat). Apc-HF: Apc ${ }^{+/ 1638 N L e p r+/+}$ mice fed a high fat diet $(60 \% \mathrm{kcal}$ fat, Apc-HF) and Apc-DB: Apc ${ }^{+/ 1638 \mathrm{NLeprdb} / \mathrm{db}}$ mice fed a low fat diet (10\% kcal fat). 
Table 3. Correlations of expression between genes involved in bacterial recognition and inflammation with genes integral to the Wnt pathway cascade.

\begin{tabular}{|c|c|c|c|}
\hline \multirow{2}{*}{$\begin{array}{l}\text { Genes involved in bacterial recognition } \\
\& \text { pro-inflammatory process }\end{array}$} & \multicolumn{3}{|c|}{ Genes within Wnt Pathway Cascade } \\
\hline & Wnts and Wnt Antagonists & Wnt Receptors and Signaling Transduction Genes & Wnt Target Genes \\
\hline MyD88 & NS & $\mathrm{LRP}^{*}, \mathrm{Apc}^{* *}, \mathrm{GSK} 3 \beta^{* *}, \beta$-catenin ${ }^{* *}, \mathrm{Akt}^{* *}$ & C-myc**, Cyclin D1*, Jnk1**, C-Jun* \\
\hline NFкB1 & SFRP5* & $\mathrm{Dvl}^{*}, \mathrm{LRP}^{* *}, \mathrm{Apc}^{* *}, \mathrm{GSK} 3 \beta^{* *}, \beta$-catenin ${ }^{* *}, \mathrm{Akt}^{* *}$ & $\begin{array}{l}\text { C-myc**, Cyclin D1**, Axin 2*, Jnk1**, } \\
\text { C-Jun** }\end{array}$ \\
\hline Nod2 & NS & NS & NS \\
\hline
\end{tabular}

${ }^{*} p<0.05 ;{ }^{* *} p<0.01$; NS: No statistically significant correlations identified.

\section{Discussion}

Although obesity is an established risk factor for the development of several malignancies $(27,28)$, including CRC (29-31), the detailed mechanisms mediating this relationship require further elucidation. We previously demonstrated that obesity elevates Wnt-signaling in the colon (10). By directly comparing two modalities by which obesity is produced the current study expands on prior observations by examining the effects produced by a high fat diet versus those produced by obesity per se (i.e.: genetically-induced obesity). Further, the present study also begins to examine the potential roles that members of the intestinal microbiome might play in this process. Findings from this study indicated that diet- and genetically-induced obesity resulted in distinct bacterial patterns, but both of them leads to an elevated state of intestinal inflammation as indicated by increased inflammatory cytokines, and in parallel with that are the alterations of the Wnt-signaling pathway and increased tumorigenesis in the obese $\mathrm{Apc}^{+/ 1638 \mathrm{~N}}$ model.

The current study demonstrated that several bacteria are differentially altered by diet- and genetically-induced obesity. Of note, the abundance of genus Turicibacter was decreased and species $F$. prausnitizii increased in the small intestine of the Apc-HF, but not the Apc-DB group (Fig. 2). In line with our observation, Everard et al showed that Turicibacter decreased markedly in mice fed a high fat diet (32). Data from a couple of studies showing a depletion of Turicibacter in animal models of inflammatory bowel disease $(33,34)$ raises the question whether this taxa might have anti-inflammatory properties. The species $F$. prausnitizii is generally considered an anti-inflammatory commensal bacterium that can secrete metabolites that block NFkB activation (35). Contrary to our expectations and findings from others $(36,37)$, an increased abundance of F. prausnitizii was observed in our study. But our data is not unique in this regard: Faval et al (38) reported that a 24-week intervention with a high saturated fat diet significantly increased F. prausnitizii in 88 subjects 'at risk' of metabolic syndrome. This inconsistency underscores the complexity of intestinal microbiota, and suggests that there may be additional as of yet unidentified factors that interact with $F$. prausnitizii in determining its effects on the intestinal environment.

A. mucinphila, E. rectale and Bifidobacterium decreased whereas Peptostreptococcus increased only in the genetically-induced Apc-DB obese group. The mucin-degrading bacterium, A.muciniphila, and butyrate-producing bacterium, E.rectale, are thought to protect against colorectal cancer and colitis $(39,40)$. Members of the Bifidobacterium genus are widely used as probiotics, and its abundance has been shown to be significantly reduced in colon cancer patients (41). Peptostreptococcus is a commensal bacterium that can cause a wide variety of infections in multiple sites, including small intestine mucosa. It is also abundant in the gut microbiota of colon cancer patients (40). The changes in these genera and species are therefore all consistent with what one might expect in the Apc-DB group, which displays enhanced tumorigenesis.

Interestingly, when we examined correlations between the altered intestinal microbiota with mouse body weight, the results showed that significant associations were mainly observed for those bacteria altered by genetic-induced obesity (Table 1). This result may simply be due to the fact that a greater magnitude of obesity was achieved with the genetically-induced obesity and therefore correlations might be easier to detect, but might also indicate that the dysbiosis observed in Apc-HF mice might be more determined by aspects of the diet rather than the obese state per se.

Despite the differences in microbial profiles between the two modes of obesity, there was a commonality of inflammation. Among the genes involved in bacterial recognition and inflammation, myeloid differentiation factor 88 (MyD88) protein--a cytoplasmic molecule that plays a central role in the immune response to bacterial cell components, including lipopolysaccharide, peptidoglycan, and lipopeptide $(25,26)$--was significantly increased in both diet- and genetically-induced obesity (Fig. 3A). MyD88 has a very complex role in tumorigenesis: its expression has often been observed to have a pro-tumorigenic effect in the liver (42) and colorectum (43), but it also assumes a tumor suppressive role in 
some inflammation-associated cancer models $(44,45)$. The findings from these studies reiterate the need for further elucidation of this gene's roles in explaining the inter-relationships between the intestinal microbiome, inflammation and tumorigenesis in the setting of obesity. No significant differences were observed in the patterns of elevated inflammatory cytokines between diet- and genetically-induced obesity. The incremental increase in TNF- $\alpha$ protein concentration across the three groups paralleled the incremental increases in both body weight and tumor incidence (20) (Fig. 3B), underscoring prior observations that have established causative links between obesity, intestinal TNF- $\alpha$, and tumorigenesis $(4,10)$.

Similarly, the expression patterns of genes in Wnt pathway were not differently altered between the diet- and genetically-induced obese groups when comparing them to the low fat group (Fig. 4B). When we correlated expressions of those significantly altered genes involved in bacterial recognition and pro-inflammatory processes $(M y D 88, N F \kappa B 1$ and Nod2) with expressions of genes within the Wnt pathway, multiple genes were identified to be significantly associated with MyD88 and NFkB1, particularly the downstream target genes and signaling transduction genes (Table 3). Our prior study (10) reported that obesity-promoted inflammation (TNF- $\alpha$ ) may phosphorylate GSK3 $\beta$ and thereby activate Wnt-signaling and increase expressions of Wnt pathway downstream genes. Therefore, it is not surprising a group of downstream target genes whose transcriptions were identified to be elevated in this study. Interestingly, elevated transcriptional levels of a number of signaling transduction genes, such as $A p c$, GSK3B and $\beta$-catenin, were observed in this study. Apc, GSK $\beta$, and 8 -catenin operate as a complex that plays a critical role in the regulation of b-catenin degradation in the Wnt pathway (46). However, evidence is limited and inconsistent regarding how a high fat diet or obesity per se regulates expression of these genes. For instance, a transcriptional upregulation of b-catenin was reported in one study in response to high fat diet (47). whereas another study showed that B-catenin protein, but not the mRNA, is elevated in genetic obesity and reduced by calorie restriction (48). Further investigation into the regulation of this complex is needed. Nevertheless, significant correlations between $M y D 88$ and $N F \kappa B 1$ with the 2 critical Wnt pathway downstream oncogenes, C-Myc and Cyclin D1, inform the potential mechanism that obesity mediates intestinal tumorigenesis via inflammation-driven activation of Wnt-signaling (Fig. $4 C)$.
This study represented a logical extension of our previous publications $(10,20)$ and added significant insights into the mechanisms mediating obesity-associated intestinal cancer. Using the $\mathrm{Apc}^{+/ 1638 \mathrm{~N}}$ mouse model, this study clearly demonstrated that the inflammation-driven Wnt-signaling serves as a critical mechanism towards enhanced intestinal cancer in the obese state. In terms of the characterization of microbiome, in this work, we particularly focused on the small intestinal microbial constitution, as opposite the colonic microbial composition, since the tumors exclusively occur in the small intestine in this mouse model and the small intestinal microbiome is more reflective of the tumor microenvironment. We demonstrated several bacterial taxa exclusively associated with the two modalities of obesity, but both leads to similar alterations of genes involved in bacterial recognition (MyD88) and inflammatory cytokines (IFN $\gamma$, IL-6 and TNF- $\alpha$ ) in parallel with alterations of genes that are integral components of the Wnt-signaling cascade in a fashion indicating its activation. In this work, we focused on inflammatory regulation of the critical Wnt pathway, alterations of which appear in up to $90 \%$ of CRC $(12,13)$. However, it is noteworthy that other pathways may also be altered, probably in a less degree, and thereby contribute to the development of CRC in the obese state. For instance, the MAPK pathway is also a critical pathway in tumorigenesis. The MAPK possesses the capability to phosphate $C-m y c$, via which it can interact with the Wnt pathway and regulate cell proliferation and apoptosis. A defect of the MAPK pathway has been demonstrated to be able to promote uncontrolled growth, a necessary step for the development of many types of cancers including CRC. The MAPK pathway also plays a critical role in the regulation of a number of the inflammatory regulators, such as NF- $\kappa \mathrm{B}$ and AP-1, and in turn leads to tumorigenesis $(49,50)$.

In summary, this study has examined how obesity alters the microenvironment of the small intestine of the $\mathrm{Apc}^{+/ 1638 \mathrm{~N}}$ mouse. We have demonstrated that, although high fat diet-induced and genetically-induced obesity alter the intestinal microbiome differently, both lead to similar states of biochemical inflammation and similar alterations in gene expression among members of the Wnt pathway towards enhancing intestinal tumorigenesis. These salient findings not only demonstrate inflammation-driven Wnt-signaling as a novel mechanism mediating obesity-associated intestinal cancer, the information provided by this work also implies that new strategies to suppress inflammation-driven Wnt-signaling can be utilized to attenuate intestinal cancer regardless the two 
modalities of obesity, but different approaches should be used if targeting to mediate gut microbiome.

\section{Supplementary Material}

Supplementary tables.

http://www.jcancer.org/v07p1780s1.pdf

\section{Acknowledgement}

This project is supported by USDA grants (2014-67017-21762 \& MAS00454 to ZL), SPHHS Deans Incentive Grant (ZL), and the Agricultural Research Service of the United States Department of Agriculture (Cooperative Agreements \#58-1950-0-014 and \#58-1950-4-003 to JBM).

\section{Author Contributions}

Conception and design of the experiments: ZL S-WC JWC JBM. Conduction of the experiments and acquisition of data: WL JWC ACP LL JL ZL. Analysis and interpretation of the data: ZL WL JWC JBM. Writing of the manuscript: ZL WL JWC JBM.

\section{Competing Interests}

The authors have declared that no competing interest exists.

\section{References}

1. Kelly T, Yang W, Chen CS, Reynolds K, He J. Global burden of obesity in 2005 and projections to 2030. Int J Obes (Lond) 2008; 32: 1431-7.

2. Finkelstein EA, Khavjou OA, Thompson H, et al. Obesity and severe obesity forecasts through 2030. Am J Prev Med 2012; 42: 563-70.

3. Calle EE, Kaaks R. Overweight, obesity and cancer: epidemiological evidence and proposed mechanisms. Nat Rev Cancer 2004; 4: 579-91.

4. Flores MB, Rocha GZ, Damas-Souza DM, et al. Obesity-Induced Increase in Tumor Necrosis Factor-alpha Leads to Development of Colon Cancer in Mice. Gastroenterology 2012; 143: 741-53 e4.

5. Gravaghi C, Bo J, Laperle $\mathrm{KM}$, et al. Obesity enhances gastrointestinal tumorigenesis in Apc-mutant mice. Int J Obes (Lond) 2008; 32: 1716-9.

6. Torre LA, Bray F, Siegel RL, Ferlay J, Lortet-Tieulent J, Jemal A. Global cancer statistics, 2012. CA Cancer J Clin 2015; 65: 87-108.

7. Siegel RL, Miller KD, Jemal A. Cancer statistics, 2015. CA Cancer J Clin 2015; 65: 5-29.

8. Ouchi N, Parker JL, Lugus JJ, Walsh K. Adipokines in inflammation and metabolic disease. Nat Rev Immunol 2011; 11: 85-97.

9. Karagiannides I, Pothoulakis C. Obesity, innate immunity and gut inflammation. Curr Opin Gastroenterol 2007; 23: 661-6.

10. Liu Z, Brooks RS, Ciappio ED, et al. Diet-induced obesity elevates colonic TNF-alpha in mice and is accompanied by an activation of Wnt signaling: a mechanism for obesity-associated colorectal cancer. J Nutr Biochem 2012; 23 : 1207-13.

11. Jain SS, Bird RP. Elevated expression of tumor necrosis factor-alpha signaling molecules in colonic tumors of Zucker obese ( $\mathrm{fa} / \mathrm{fa}$ ) rats. Int J Cancer 2010; 127 : 2042-50.

12. Taketo MM. Shutting down Wnt signal-activated cancer. Nat Genet 2004; 36: 320-2.

13. Network CGA. Comprehensive molecular characterization of human colon and rectal cancer. Nature 2012; 487: 330-7.

14. Schwabe RF, Jobin C. The microbiome and cancer. Nat Rev Cancer 2013; 13: 800-12.

15. Turnbaugh PJ, Backhed F, Fulton L, Gordon JI. Diet-induced obesity is linked to marked but reversible alterations in the mouse distal gut microbiome. Cell Host Microbe 2008; 3: 213-23.

16. Turnbaugh PJ, Ley RE, Mahowald MA, Magrini V, Mardis ER, Gordon JI. An obesity-associated gut microbiome with increased capacity for energy harvest. Nature 2006; 444: 1027-31.

17. Kamada N, Seo SU, Chen GY, Nunez G. Role of the gut microbiota in immunity and inflammatory disease. Nat Rev Immunol 2013; 13: 321-35.

18. Liu Z, Ciappio ED, Crott JW, et al. Combined inadequacies of multiple B vitamins amplify colonic Wnt signaling and promote intestinal tumorigenesis in BAT-LacZxApc1638N mice. FASEB J 2011; 25: 3136-45.
19. Huffman DM, Augenlicht LH, Zhang $X$ et al. Abdominal obesity, independent from caloric intake, accounts for the development of intestinal tumors in Apc(1638N/+) female mice. Cancer Prev Res (Phila) 2013; 6: 177-87.

20. Pfalzer AC, Nesbeth PD, Parnell LD, et al. Diet- and Genetically-Induced Obesity Differentially Affect the Fecal Microbiome and Metabolome in Apc1638N Mice. PLoS One 2015; 10: e0135758.

21. Hummel KP, Dickie MM, Coleman DL. Diabetes, a new mutation in the mouse. Science 1966; 153: 1127-8.

22. Fodde R, Edelmann W, Yang K, et al. A targeted chain-termination mutation in the mouse Apc gene results in multiple intestinal tumors. Proc Natl Acad Sci U S A 1994; 91: 8969-73.

23. Borges-Canha M, Portela-Cidade JP, Dinis-Ribeiro M, Leite-Moreira AF, Pimentel-Nunes P. Role of colonic microbiota in colorectal carcinogenesis: A systematic review. Rev Esp Enferm Dig 2015; 107.

24. Deng W, Wang Y, Liu Z, Cheng H, Xue Y. HemI: a toolkit for illustrating heatmaps. PLoS One 2014; 9: e111988.

25. Villano JS, Rong F, Cooper TK. Bacterial infections in Myd88-deficient mice. Comp Med 2014; 64: 110-4

26. Kawai T, Adachi O, Ogawa T, Takeda K, Akira S. Unresponsiveness of MyD88-deficient mice to endotoxin. Immunity 1999; 11: 115-22.

27. Bianchini F, Kaaks R, Vainio H. Overweight, obesity, and cancer risk. Lancet Oncol 2002; 3: 565-74.

28. Calle EE, Rodriguez C, Walker-Thurmond K, Thun MJ. Overweight, obesity, and mortality from cancer in a prospectively studied cohort of U.S. adults. N Engl J Med 2003; 348: 1625-38.

29. Murphy TK, Calle EE, Rodriguez C, Kahn HS, Thun MJ. Body mass index and colon cancer mortality in a large prospective study. Am J Epidemiol 2000; 152: 847-54.

30. Thun MJ, Calle EE, Namboodiri MM, et al. Risk factors for fatal colon cancer in a large prospective study. J Natl Cancer Inst 1992; 84: 1491-500.

31. Moghaddam AA, Woodward M, Huxley R. Obesity and risk of colorectal cancer: a meta-analysis of 31 studies with 70,000 events. Cancer Epidemiol Biomarkers Prev 2007; 16: 2533-47.

32. Everard A, Lazarevic V, Gaia N, et al. Microbiome of prebiotic-treated mice reveals novel targets involved in host response during obesity. ISME J 2014; 8: 2116-30.

33. Jones-Hall YL, Kozik A, Nakatsu C. Ablation of tumor necrosis factor is associated with decreased inflammation and alterations of the microbiota in a mouse model of inflammatory bowel disease. PLoS One 2015; 10: e0119441.

34. Rossi G, Pengo G, Caldin M, et al. Comparison of microbiological, histological, and immunomodulatory parameters in response to treatment with either combination therapy with prednisone and metronidazole or probiotic VSL\#3 strains in dogs with idiopathic inflammatory bowel disease. PLoS One 2014; 9: e94699.

35. Sokol H, Pigneur B, Watterlot L, et al. Faecalibacterium prausnitzii is an anti-inflammatory commensal bacterium identified by gut microbiota analysis of Crohn disease patients. Proc Natl Acad Sci U S A 2008; 105: 16731-6.

36. Remely M, Tesar I, Hippe B, Gnauer S, Rust P, Haslberger AG. Gut microbiota composition correlates with changes in body fat content due to weight loss. Benef Microbes 2015; 6: 431-9.

37. Stenman LK, Burcelin R, Lahtinen S. Establishing a causal link between gut microbes, body weight gain and glucose metabolism in humans - towards treatment with probiotics. Benef Microbes 2015: 1-12.

38. Fava F, Gitau R, Griffin BA, Gibson GR, Tuohy KM, Lovegrove JA. The type and quantity of dietary fat and carbohydrate alter faecal microbiome and short-chain fatty acid excretion in a metabolic syndrome 'at-risk' population. Int J Obes (Lond) 2013; 37: 216-23

39. Everard A, Belzer C, Geurts L, et al. Cross-talk between Akkermansia muciniphila and intestinal epithelium controls diet-induced obesity. Proc Natl Acad Sci U S A 2013; 110: 9066-71.

40. Wang T, Cai G, Qiu Y, et al. Structural segregation of gut microbiota between colorectal cancer patients and healthy volunteers. ISME J 2012; 6: 320-9.

41. Singh J, Rivenson A, Tomita M, Shimamura S, Ishibashi N, Reddy BS, Bifidobacterium longum, a lactic acid-producing intestinal bacterium inhibits colon cancer and modulates the intermediate biomarkers of colon carcinogenesis. Carcinogenesis 1997; 18: 833-41.

42. Naugler WE, Sakurai T, Kim S, et al. Gender disparity in liver cancer due to sex differences in MyD88-dependent IL-6 production. Science 2007; 317: 121-4.

43. Uronis JM, Muhlbauer $\mathrm{M}$, Herfarth $\mathrm{HH}$, Rubinas TC, Jones GS, Jobin C Modulation of the intestinal microbiota alters colitis-associated colorectal cancer susceptibility. PLoS One 2009; 4: e6026.

44. Ochi A, Nguyen AH, Bedrosian AS, et al. MyD88 inhibition amplifies dendritic cell capacity to promote pancreatic carcinogenesis via Th2 cells. J Exp Med 2012; 209: 1671-87.

45. Salcedo $\mathrm{R}$, Worschech $\mathrm{A}$, Cardone $\mathrm{M}$, et al. MyD88-mediated signaling prevents development of adenocarcinomas of the colon: role of interleukin 18 . J Exp Med 2010; 207: 1625-36.

46. Clevers H. Wnt/beta-catenin signaling in development and disease. Cell 2006; 127: 469-80.

47. Waller-Evans H, Hue C, Fearnside J, et al. Nutrigenomics of high fat diet induced obesity in mice suggests relationships between susceptibility to fatty liver disease and the proteasome. PLoS One 2013; 8: e82825.

48. Mao J, Hu X, Xiao Y, et al. Overnutrition stimulates intestinal epithelium proliferation through beta-catenin signaling in obese mice. Diabetes 2013; 62: $3736-46$ 
49. Sun P, Zhou K, Wang S, et al. Involvement of MAPK/NF-kappaB signaling in the activation of the cholinergic anti-inflammatory pathway in experimental colitis by chronic vagus nerve stimulation. PLoS One 2013; 8: e69424.

50. Karin M. The regulation of AP-1 activity by mitogen-activated protein kinases. J Biol Chem 1995; 270: 16483-6. 